\title{
Un monde rêvé : la France dans la revue Xinyue
} (1928-1933)

A dreamed world: France in Xinjue Journal (1928-1933))

Jacqueline ESTRAN

\section{(2) OpenEdition}

Journals

Édition électronique

URL : http://journals.openedition.org/transtexts/197

DOI : $10.4000 /$ transtexts. 197

ISSN : 2105-2549

Éditeur

Gregory B. Lee

Édition imprimée

Date de publication : 1 mai 2006

Pagination : 167-181

ISSN : 1771-2084

\section{Référence électronique}

Jacqueline ESTRAN, « Un monde rêvé : la France dans la revue Xinyue (1928-1933) », Transtext(e)s

Transcultures 跨文本跨文化 [En ligne], 1 | 2006, mis en ligne le 13 septembre 2009, consulté le 02 mai 2019. URL : http://journals.openedition.org/transtexts/197 ; DOI : 10.4000/transtexts. 197 


\title{
Un monde rêvé : la France dans la revue Xinyue (1928-1933) $B Y$ Jacqueline Estran
}

\begin{abstract}
La revue Xinyue fait partie des trois revues ayant joué un rôle de leader dans le débat d'idées en Chine dans les années vingt/trente. Elle se positionne sur le plan idéologique / politique entre Parti communiste et Parti Nationaliste et vise, sur le plan culturel, à réaliser une synthèse entre la tradition chinoise et les civilisations occidentales. Les articles publiés traitent abondamment du monde anglophone - « anglophilie » qui s'explique par la formation des principaux contributeurs de la revue - mais s'intéressent aussi à la France, France qui est une référence récurrente pour les intellectuels chinois de la première moitié du xxe siècle. Les textes qui lui sont consacrés dans la revue, qu'ils soient à contenu littéraire, philosophique, politique ou social, permettent de dresser une image de la France, de laquelle il ressort que la France est plus un miroir reflétant la Chine qu'une entité autonome étudiée pour elle-même.
\end{abstract}

Xinyue is one of the three periodicals which played a leading role in the intellectual debates that took place in the 1920s and 1930s China. It was ideologically positioned between the Communist and the Nationalist parties, and aimed at a cultural synthesis of the Chinese tradition and Western civilization. The articles published dealt extensively with the English-speaking world, which was a consequence of the educational background of the main contributors; yet they also spoke about France, a constant reference for the Chinese intellectuals in the first half of the twentieth century. The writings relative to France, whether discussing literary, philosophical, political or social questions, conjured up a picture which was more a mirror reflecting China than an autonomous entity examined for its own sake.

《新月》是二十世纪二三十年代领导中国思潮的三本杂志之一。在意识形态与 政治方面，它定位于共产党与国民党之间，在文化方面，它力求实现中国传统 与西方文明间的综合。《新月》发表的文章主要涉及英语世界一一杂志的主要 拟稿人队伍认为它是“亲英的”一然而，它对法国也抱有一定兴趣。对于二 十世纪上半叶的中国知识分子来说，法国是一个经常提及的话题。《新月》杂 志中的文章，是些关于文学、哲学、政治或社会的内容，它们使得建树一种关 于法国的形象成为可能。由此形象可见, 法国更多地是折射中国的一面镜子, 而并非是一个对其自身而言被研究的自治实体。 


\section{Introduction}

Le groupe qui s'est constitué autour de la revue Xinyue 新月(Croissant / Nouvelle lune) et qui comporte quelques-unes des grandes figures intellectuelles chinoises de la première moitié du XXe siècle - tels Hu Shi 胡適 (1891-1962), Wen Yiduo 聞一多 (1899-1946), Xu Zhimo 徐志摩 (1897-1931), Liang Shiqiu 梁實秋 (1902-1987) ou Shen Congwen 沈從文 (1902-1988) - a joué un rôle de premier plan, que ce soit dans le débat politique ou les polémiques littéraires. ${ }^{1} \mathrm{Et}$, au-delà de son influence immédiate sur des questions cruciales pour la fondation de la nouvelle Chine qui tente de se former alors (comme la question des droits de l'homme ou celle de la mise en place d'une nouvelle constitution), c'est aussi tout un ensemble de jeunes intellectuels qui sont influencés par Xinyue dans le domaine de la création littéraire (roman et poésie) et de la recherche et par lesquels son action se poursuit sur un plan plus général, par une «attitude» particulière vis-à-vis du monde et de la création

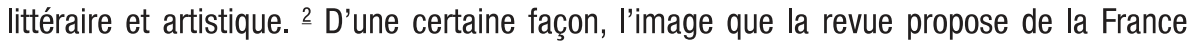
témoigne aussi de cette attitude.

L'analyse qui suit dressera cette image à partir d'un ensemble de textes qui se compose de : traductions de textes (littéraires ou non), comptes rendus d'ouvrages (essentiellement littéraires), et essais (qui portent sur la littérature, la philosophie, l'histoire et la société françaises). ${ }^{-}$

Sur le plan thématique, quatre axes se dégagent:

- une France philosophique, avec la philosophie du XVIII siècle, présentée surtout pour ses positions anti-cartésiennes et ses conceptions de la morale. Cet axe appelle surtout une interprétation par rapport à la période et au milieu contemporains à la revue et aux auteurs. Les idées soulevées dans ces textes font en effet apparaitre que le sujet n'est pas vraiment

\footnotetext{
1 Hu Shi 胡適 symbolise la révolution littéraire chinoise du 4 mai. Wen Yiduo 聞一多 et Xu Zhimo 徐志摩ont joué un rôle décisif dans le devenir de la poésie chinoise moderne tant sur le plan théorique que créatif. Liang Shiqiu梁實秋 a lancé les plus importantes polémiques du domaine littéraire de la période, en particulier en ce qui concerne la «litérature de classe». Shen Congwen 沈從文 s'est illustré dans l'écriture romanesque. Citons encore : Yu Shangyuan 余上沅 qui a ceuvré pour le théâtre chinois moderne au travers de la fondation de la première école publique de théâtre parlé (huaju) en Chine ; Rao Mengkan 饒孟㑆 et Chen Mengjia 陳夢家 en poésie ; Liang Yuchun 梁遇春 et Ye Gongchao 葉 公超 dans le domaine de la critique littéraire ainsi que Luo Longji 羅隆基 et Pan Guangdan 潘光旦 qui représentent le versant social de la revue avec des articles politiques et sociologiques, 2 Sur la place de Hu Shi et Xinyue dans la débat politique des années vingt, voir, par exemple : Marie-Claire Bergère, Lucien Bianco et Jürgen Domes, La Chine au XXe siècle - D'une révolution à l'autre 1895-1949, Paris, Fayard, 1989, pp. 332-337. 3 L'ensemble des articles traitant de la France a été sélectionné à partir de l'index thématique de la revue réalisé par l'auteur. V. J. Estran, La revue Xinyue (1928-1933) : sa contribution à la littérature chinoise moderne, (thèse de doctorat), Paris, INALCO, 2000.
} 
celui proposé, l'information est anecdotique et les idées évoquées renvoient à la Chine de l'époque, à ses débats, ses doutes et ses craintes.

- une France politique, avec la Révolution française, et, en particulier, la présentation de trois de ses principaux protagonistes : Marat, Danton et Robespierre. On peut faire la même remarque que pour l'axe précédent et rajouter que la France apparaît là comme un lieu mythique où se déroulent des événements terribles ou curieux, liés à la nature particulière de ses habitants.

- une France littéraire. Cet axe fait également référence aux discours et aux débats de l'époque en Chine mais s'inscrit de façon plus profonde dans la réflexion sur la littérature des contributeurs de la revue.

- et une France scientifique, monde de la recherche, avec des textes sur la sinologie française. Cet axe s'intéresse aux interactions entre France et Chine, par le biais d'une présentation de la recherche menée en France sur la Chine.

Au fil de ces présentations, se glissent aussi des remarques ou des allusions sur les Français en tant que peuple qui feront l'objet d'une cinquième partie.

\section{Une France philosophique}

Dans le domaine philosophique, ce sont trois articles qui sont consacrés à la France ou l'évoquent, en soixante pages environ. Deux de ces textes sont de la plume de Peng Jixiang 彭基相, le troisième est une traduction réalisée à partir de l'anglais par Ruan Ziwei 阮子蔚 . Peng Jixiang, qui traite de divers aspects de la philosophie européenne dans la revue (I-7, $8,9,10,12$ ), est issu de la sphère francophone; il a étudié à Beida puis en France où il a été en contact avec Wen Jiasi, frère de Wen Yiduo et sa première contribution à Xinyue est une correspondance envoyée de Paris. ${ }^{4} \mid I$ apporte une touche philosophique à la revue dans laquelle il publie des critiques d'ouvrages ou des essais. Des textes de Peng Jixiang et Ruan Ziwei dont il va être question, deux idées fortes ressortent : la justification des emprunts à l'Occident et une volonté de contrebalancer l'influence soviétique.

Le premier texte de Peng Jixiang traite de la philosophie française du XVIIle siècle et commence en annonçant que le XVIII français fut un siècle de renversement des idoles, un siècle de destruction, exactement dans les termes employés à l'époque pour rejeter la tradition philosophique chinoise. .5 Avant d'aborder à proprement parler son sujet, l'auteur précise que « renverser des idoles n'étant pas chose facile, la France a fait appel à l'étranger, et plus précisément aux Anglais, pour l'aider dans cette tâche $» . \underline{6}$ Concrètement, ce sont Bacon (pour la méthode), Newton (pour la nature) et Locke (pour la psychologie) qui ont servi de support à la remise en question de la pensée de Descartes, symbole du XVII siècle et objet des critiques du siècle suivant. Ce qui paraît dans ce contexte faire immédiatement référence au débat d'idées en Chine, c'est l'appel à l'étranger pour revoir ses positions, appel qui est ici longuement justifié par l'auteur au nom du progrès de la science et de la connaissance. Depuis 1860, la question de savoir dans quelle mesure utiliser les connaissances et la pensée de l'Occident est régulièrement soulevée, pour être explicitée et justifiée, d'abord par des

4 Peng Jixiang 彭基相, «Bali tongxin »巴黎通信 [Correspondance de Paris], I.6. 5 Peng Jixiang, « Faguo shiba shiji de zhexue »法國十八世紀的哲學 [La philosophie française du XVIII siècle], I.7.6 Peng, «Bali tongxin », p. 1. 
fonctionnaires du gouvernement impérial des Qing, sur un plan purement technique afin de se défendre des puissances occidentales, puis, par vagues successives, sur le fond par les lettrés progressistes chinois après l'échec de transferts purement technologiques. ${ }^{?}$ Admise dans les milieux progressistes des années dix, cette approche a été ébranlée par la trahison des puissances occidentales lors de la signature du traité de Versailles en 1919.. Peng Jixiang la reprend pour la fonder en la plaçant sur un terrain apparemment neutre sur le plan idéologique par rapport au contexte chinois : les rapports entre la France et l'Angleterre. La France ayant eu à bénéficier de ses échanges avec l'Angleterre, pourquoi la Chine n'en feraitelle pas autant? II faut, par ailleurs, noter que, consciemment ou non, l'auteur propose ici un cas où les Anglais sont un exemple, l'Angleterre un vivier potentiel d'idées intéressantes, ce qui semble pouvoir être interprété d'une part, comme un appel à chercher des idées du côté du monde anglophone et, d'autre part, comme une volonté de contrebalancer l'influence soviétique, grandissante - pour ne pas dire plus - à la fin des années vingt sur le territoire chinois.

Un autre texte - traduit celui-ci par Ruan Ziwei - reprend la première de ces idées. 9 Plaçant Voltaire parmi les dix plus grands penseurs du monde, il insiste sur le fait qu'emprunter n'enlève rien à l'intérêt ou à la valeur d'une pensée. L'approche est plus radicale puisque l'auteur ajoute qu'il est extrêmement rare d'arriver à créer véritablement et que, dans le meilleur des cas, en général, la création se contente de modifier la forme et porte rarement sur le fond, vivant là d'emprunts. Voltaire apparaît comme exemplaire, en ce sens qu'il a ouvert la voie à ses successeurs - en empruntant - et montré ainsi le chemin de la véritable liberté d'esprit.

Le second texte de Peng Jixiang, consacré à la morale, vient préciser le contexte dans lequel le renversement des idoles peut se faire. ${ }^{10}$ Prenant toujours pour point de départ la philosophie française du XVIII e siècle, il insiste sur l'importance de la morale pour la société, tout pouvant être renversé, voire supprimé, sauf la morale. Les deux positions présentées (Rousseau et D'Holbach) sont rejetées, l'auteur défendant une morale basée sur la vie pratique à même d'être respectée et mise en pratique toujours et par tous, rejoignant l'approche pragmatiste de Hu Shi. Au-delà du fait qu'il met en scène deux penseurs français, ce texte s'inscrit dans l'un des plus importants débats idéologiques et culturels de l'époque, en Chine comme à l'étranger.

7 Voir Ying-Yee Kuo et Kwang-Ching Lu, "Self-strengthening: the Pursuit of Western Technology" in J. K. Fairbank (ed.), The Cambridge History of China, vol, 10 (Late Ch'ing 1800-1911 - Part 1), Cambridge, Cambridge University Press, 1978, pp. 491-542. 8 Traité qui transférait de l'Allemagne au Japon les territoires occupés par celui-ci en Chine, sans que le gouvernement ni le peuple chinois n'aient été consultés. 9 Will Durant, "Shijie shi da sixiangjia " 世界十大思想家 [Dix maîtres à penser], trad. par Ruan Ziwei 阮子蔚, III.9. Les autres penseurs présentés dans cet article sont : Confucius, Platon, Aristote, Saint Thomas d'Aquin, Copernic, Francis Bacon, Isaac Newton, Emmanuel Kant et Charles Darwin. 10 Peng Jixiang, «Faguo shiba shiji de daode guannian »法國十八世紀的 道德觀念 [Les conceptions de la morale dans la France du XVIIII siècle], I.8. Ce texte est présenté comme une suite du texte de Peng Jixiang cité précédemment. 


\section{Une France politique}

La France apparaît également comme référence par rapport au débat politique chinois, au travers d'un ensemble de trois textes, totalisant une quarantaine de pages, tous rédigés par Yu Nanqiu. ${ }^{11}$ Celui-ci a étudié à l'institut Qinghua de 1914 à 1921, puis aux Etats-Unis pendant huit ans. Historien, il a enseigné par la suite, notamment à l'Université de Fudan où ont également enseigné d'autres collaborateurs de la revue Xinyue.

L'image proposée s'organise autour de trois figures : Robespierre, Marat et Danton, trois figures qui deviennent sous la plume de Yu Nanqiu des références pour la Chine des années vingt / trente. On peut certainement rattacher au goût pour l'histoire du peuple chinois l'intérêt manifesté pour les personnages historiques, et ce de quel que pays qu'ils soient, mais la France occupe à cet égard une position privilégiée, vraisemblablement liée au prestige de la Révolution française et à la proclamation des Droits de l'homme. Napoléon $1^{\mathrm{er}}$, qui fait partie des référents historiques en Chine, est également présent dans la revue mais pour une nouvelle et non pas en tant que personnalité politique. ${ }^{12}$

Chacune des figures présentées permet de mettre en question le rôle de l'homme politique dans la société, de discuter de ses droits, de ses devoirs ou de ses abus. Robespierre est présenté comme un autocrate, responsable du déroulement sanglant de la Révolution. Dans ce texte, Yu Nanqiu insiste sur les dangers qu'il y a à placer tous les pouvoirs dans les mains d'un seul homme et sur la responsabilité de l'individu dans le cours des événements, notamment lorsqu'il s'agit de mouvements progressistes. La Révolution française - qui aurait pu être une histoire d'hommes et d'idées - apparaît comme pervertie par Robespierre, qui en a fait une histoire de massacres. Comparativement, Marat est vu comme un héros, solitaire, défenseur du peuple, ne voulant pas du pouvoir et ne craignant pas les puissants, plaçant audessus de tout sa liberté d'expression et donnant la parole à ceux qui ne l'ont pas. Prêt à se sacrifier, il est perçu comme victime de son idéalisme. L'auteur le qualifie de vrai « dirigeant révolutionnaire " parce qu'il a approuvé le massacre de septembre 1792, présenté comme indispensable. L'article est tellement élogieux qu'il est difficile de savoir si l'auteur est ironique ou s'il approuve simplement qu'en une époque troublée, des têtes doivent tomber. Pour information sur le contexte, il faut rappeler que les massacres font alors partie, sinon du quotidien, du moins de la réalité chinoise, on peut ainsi mentionner l'épuration subie par les sympathisants communistes en avril 1927 dans la ville de Shanghai, épuration qui a fait des milliers de victimes. ${ }^{13}$ Par ailleurs, l'auteur fait une référence directe au contexte chinois puisqu'il dit explicitement que Marat n'était d'aucun parti mais que s'il avait été un contemporain, il aurait sûrement été communiste (ce qui semble renvoyer au fait que Marat a été assassiné dans sa baignoire comme les communistes sont morts dans un bain de sang

11 Yu Nanqiu 余楠秋(1897-1968), «Luobosipi'er »羅伯斯庇爾[Robespierre], III.1, «Dantang » 丹塘 [Danton] , III.5/6, « Mala"; 馬拉[Marat], II.9. 12 Voir infra note 26,13 Voir Jerome Ch'en, "The Chinese Communist Movement to 1927", Martin C. Wilbur, "The Nationalist Revolution from Canton to Nanking 1923-1928" in D. Twitchett \& J. K. Fairbank (eds.), The Cambridge History of China, vol. 12 (Republican China - Part 1), Cambridge, Cambridge University Press, 1983. 
en 1927). $\stackrel{14}{ }$ Danton est encore plus parfait que Marat et dépasse largement Robespierre et Napoléon : Napoléon a réussi par chance, parce qu'il a su saisir des opportunités, tandis que Robespierre était un homme quelconque essentiellement préoccupé par son intérêt personnel. Danton est, lui, véritablement au service de son pays : il est capable d'en comprendre les besoins ainsi que ceux de ses compatriotes, d'éprouver de la compassion pour les ouvriers et de les défendre. L'auteur lui reconnaît tout de même une responsabilité dans la mise en place de la cour de justice révolutionnaire et en tant que défenseur de la peine de mort. Néanmoins, la cruauté dont semblent témoigner ces deux institutions est justifiée par le caractère emporté de Danton, en cela semblable à ses compatriotes. Les Français sont décrits dans ce contexte comme des êtres libres, ayant l'esprit vif, sachant parler, tenaces, aimant la plaisanterie, nationalistes par habitude et, dernière caractéristique, se mettant facilement en colère. Danton n'est pas critiqué, il est présenté comme un révolutionnaire exemplaire. L'élément sur lequel Yu insiste le plus est sa capacité à se mettre au service de son pays. Appel à peine voilé au sacrifice pour la cause nationale qui préoccupe l'élite intellectuelle chinoise de façon un peu désespérante à l'époque, après les nombreuses tentatives de réforme sans résultat depuis la fin du XIXe siècle.

Si la France apparaît comme un lieu où se déroulent des événements hors normes, ceux-ci sont justifiés par les circonstances et , somme toute, ils ne sont peut-être pas si dépaysants pour un ressortissant chinois du début du XXe siècle. En lisant l'histoire de France comme il lirait l'histoire chinoise, c'est-à-dire en se servant des faits du passé pour transmettre un message à ses contemporains, Yu Nanqiu intègre la France à l'univers chinois, comme on le ferait d'une contrée encore peu familière et pourtant proche.

\section{Une France littéraire}

L'introduction de la littérature étrangère en Chine s'inscrit dans un contexte bien déterminé : il s'agissait de présenter des œuvres et des mouvements littéraires afin d'aider à la mise en place d'une nouvelle littérature chinoise, littérature alors à la recherche de nouvelles formes et de nouveaux contenus, rompant de façon plus ou moins radicale avec son passé et sa tradition..$^{15}$

La littérature étrangère représente un quart des textes de la revue et, parmi ceux-ci, 1/6 - soit environ 17\% - sont consacrés à la France, (c'est-à-dire 26 textes), le reste traitant essentiellement du monde anglophone (Royaume-Uni, Irlande et Etats-Unis) et, dans une moindre mesure, du Japon, de la Russie et de l'Allemagne. $\frac{16}{2}$ Le panorama des auteurs présentés et traduits dans la revue concerne tant les genres du théâtre que ceux du roman et de la poésie.

Le théâtre occupe une toute petite place avec la traduction d'une pièce de Tristan Bernard par Li Qingya 17- mais, toutes langues étrangères confondues, neuf pièces seulement sont

\footnotetext{
14 Les collaborateurs de la revue Xinyue, qui étaient pour la plupart installés à Pékin, ont quilté cette ville pour Shanghai à partir de l'été 1926, en raison de l'insécurité grandissante en Chine du nord. Ils fondent à Shanghai la maison d'édition Xinyue en 1927 avant de lancer la revue en mars 1928. Ils sont donc les témoins directs des événements de Shanghai au cours de cette période et, sans être communistes, comptent des amis parmi les militants communistes. 15 Voir par exemple, Merle Goldman, Modern Chinese Literature in the May Fourth Era, Cambridge (Mass.) / London, Harvard University Press, 1977. 16 C'est-à-dire cent soixante cinq textes sur un total de sept cents environ, publiés sur 43 numéros et un peu plus de cinq ans. 17 Li Qingya 李青崖 (1884-1969), «Qiuhunzhe 》求婚者 [Le prétendant], II.5/6. Li est un spécialiste de la France qui publie déjà depuis de nombreuses années des traductions à partir du français, en particulier depuis 1923 dans le Xiaoshuo yuebao 小说月 報 (Maupassant, Zola, Barbusse). II publie au total quatre traductions à partir du français dans la revue Xinyue, c'est-à-dire la majorité des textes relevant du roman.
} 
traduites dans la revue. ${ }^{18}$ Cette pièce en un acte propose une vision satirique de la petite bourgeoisie française. Un homme qui doit recevoir la visite d'un prétendant fort bien doté pour sa fille se prend à douter : et s'il s'agissait d'un espion du fisc, chargé de vérifier l'exactitude de ses déclarations? Pour parer à cette éventualité, il dépeint sa situation financière sous le jour le plus sombre, ce qui fait, comme on pouvait le prévoir, fuir le prétendant. L'histoire se termine pourtant bien, puisque le prétendant officiel avait amené un ami, poète fils d'un grand industriel, qui cherchait, lui, à épouser une jeune fille pauvre et s'est épris de la jeune fille pendant que le père discutait affaires avec le prétendant officiel. Le tableau très cru qui est fait des mariages d'intérêt (où, en quelques phrases, on dépeint sa situation financière afin de voir si elle s'accorde à celle du parti opposé, sans prendre en compte les sentiments d'aucun des jeunes gens) renvoie à la pratique chinoise encore en vigueur à l'époque, dont la jeune génération intellectuelle - qui revendique alors la liberté d'aimer - aimerait bien se débarrasser. ${ }^{19}$ Un parallèle est donc établi entre les deux pays et la France ne sert pas de modèle puisque la situation n'y semble pas plus avancée qu'en Chine. En revanche, la façon d'envisager les choses permet de les dédramatiser et, surtout, cette pratique est tournée en dérision puisque c'est une peur vraisemblablement infondée qui fait refuser au père un parti intéressant pour sa fille et que le prétendant officiel est présenté pour ce qu'il est, c'est-àdire un homme d'affaires préoccupé surtout de faire fructifier sa fortune, tandis que la jeune fille et le poète trouvent seuls une voie de sortie grâce à leurs sentiments.

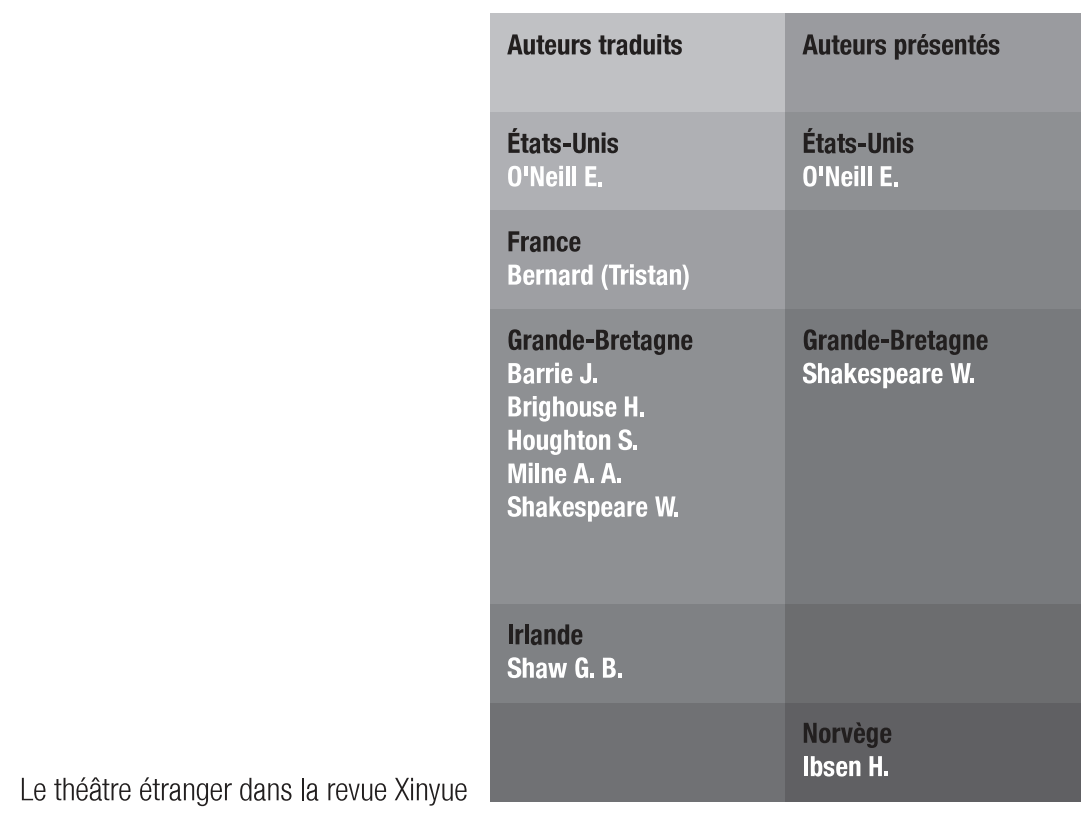

18 Outre la pièce de Tristan Bernard, Le prétendant, on trouve dans la revue la traduction des pièces suivantes: The Dear Departed de S. Houghton par Gu Zhongyi 顧仲蕬, The Twelve Pound Clock de James Barrie par Gu Zhongyi, The Artist, a Duologue de A. A. Milne par Yu Gengyu于慻虞, Man and Superman: A Comedy and a Philosophy de G. B. Shaw par Xiong Shiyi 熊式式, Followers : A Cranford Sketch de H. Brighouse par Xiong Zhengqin 熊正瑾, The Long Voyage Home (1919) d'Eugène O'Neill par Ma Yanxiang 馬顔祥, Beyond the Horizon d'Eugène O'Neill par Gu Zhongyi, des extraits de Roméo et Juliette par Xu Zhimo (extraits publiés à titre posthume.). 19 La liberté d'aimer (lian'ai ziyou 戀愛自由) est l'un des mots d'ordre de la génération qui a une vingtaine d'années au moment du 4 mai 1919. 
Dans le domaine du roman, concernant la France, on trouve des traductions et des comptes rendus d'ouvrages mais aucun essai. $\underline{20}$

Au total, vingt-cinq nouvelles ou extraits de romans sont traduits dans la revue, représentant dix-sept auteurs. Quatre sont français, deux japonais, tous les autres anglais, américains ou intégrés au monde anglophone. Pour la France, les textes traduits ressortent de registres extrêmement variés avec : Abélard et Héloïse, Marguerite de Navarre, Voltaire, Charles Nodier et Villiers de I'Isle-Adam. ${ }^{21}$ La littérature française est largement représentée en Chine à cette époque. Une comparaison avec les traductions d'œuvres romanesques publiées dans le Xiaoshuo yuebao 小说月報 [Mensuel du roman] montre que, dans celui-ci, la France occupe la deuxième place, juste après la Russie, par le nombre de ses traductions, de 1921 à 1925.른 Sa position rétrograde ensuite légèrement au profit du Japon et d'autres pays d'Europe mais elle demeure très présente par le nombre d'articles et d'ouvrages qui lui sont consacrés..$^{23} \mathrm{La}$ présence de la France apparaît donc comme représentative d'une tendance répandue dans le monde littéraire chinois des années vingt et du début des années trente, tandis que les romanciers anglophones, qui dominent dans Xinyue, sont sur-représentés par rapport à ce qui se fait dans les autres revues. 24

La revue propose également de nombreux comptes rendus d'œuvres littéraires. Dans certains cas, ces comptes rendus sont rédigés à partir d'ouvrages traduits en chinois, dans d'autres, à partir de la langue originale ou d'une traduction en anglais. II s'agit, en principe, d'œuvres qui sont d'actualité, soit dans un pays anglophone, soit en France. ${ }^{25}$ L'objectif peut être simplement de présenter l'œuvre mais le compte rendu fournit aussi souvent l'occasion de faire un commentaire sur son contenu. L'ensemble est assez hétéroclite comme il apparaît ci-dessous.

Ainsi, c'est dans ce contexte que Liang Yuchun rend compte de la découverte toute récente d'une nouvelle inédite de Napoléon $1^{\text {er }}$ (1769-1821), Clisson et Eugénie, relatée par la Revue des deux mondes. ${ }^{(26)}$ Dans cette brève présentation, il retrace l'histoire de Clisson et Eugénie, dont les critiques s'accordent généralement à dire que le personnage masculin incarne Napoléon lui-même. Liang en retient surtout l'éclairage apporté sur la personnalité intime de

\footnotetext{
20 Tous les essais sont consacrés au monde anglophone : Katherine Mansfield est présentée par Chen Xiying 陳西瀅 , Walter Scott par Fei Jianzhao 費鑑照, Thomas Hardy par Xu Zhimo et Guo Yousho 郭有守. Ye Gongchao 葉公超 propose, pour sa part, un essai sur le mouvement réaliste anglais. 21 Abélard et Héloïse : Lettres (1 et 2) traduites par Liang Shiqiu, Xinyue, I.8 ; M. de Navarre : "Opinion d'un Castillanais », extrait de l'Heptaméron traduit par Li Qingya, IV.1 ; Ch. Nodier : Jean-François les bas-bleus, traduit par Li Qingya, III.12 ; Voltaire : Le blanc et le noir, traduit par Li Qingya, III.11; Villiers de I'Isle-Adam: Les demoiselles de Bienfilatre, traduit par Xiao Shijun 萧石君, II.1, 22 Comparaison réalisée d'après un index du Xiaoshuo yuebao (Xiaoshuo yuebao suoyin (1921-1931)小说月報索引1921 -1931, Shumu wenxian chubanshe, Beijing, 1984). Jin Siyan, dans l'étude qu'elle réalise sur la période 1921-1925, place la France en troisième position derrière I'Inde et la Russie mais elle prend en compte tous les textes littéraires traduits et pas seulement les romans ou nouvelles. Voir Jin Siyan, La Métamorphose des images poétiques 1915-1932 : des symbolistes français aux symbolistes chinois, Dortmund, Projekt VIg., 1997, p. 235, 23 Voir Zhongguo xin wenxue daxi 1917-27中國新文學大系1917-1927 [Anthologie de la nouvelle littérature chinoise, 1917-1927], t. 10, Taipei, Yeqiang chubanshe, 1990 (rééd.), p. 273. Parmi les ouvrages retenus sur cette période par les compilateurs de l'anthologie, on trouve trois histoires de la littérature française pour une histoire de la littérature allemande, deux de la littérature russe, une italienne et une japonaise. 24 En effet, dans le Xiaoshuo yuebao, ce sont, de 1928 à 1931 , dans le meilleur des cas, quatre traductions qui sont publiées chaque année (Grande-Bretagne et États-Unis confondus) et, dans le pire, aucune (1928). II s'agit là d'une tendance constante au cours des dix ans de parution du Xiaoshuo yuebao, avec une exception pour 1927, année au cours de laquelle sept textes sont publiés. 25 On peut estimer que le décalage entre ce qui se passe dans les pays occidentaux et la retranscription qui en est faite dans la revue Xinyue n'excède pas un an. La date de publication de l'œuvre à l'étranger est en général mentionnée. 26 Liang Yuchun 梁 遇春 (1906-1932), «Xin faxian de Napolun de xiaoshuo»新發現的拿破侖的小說 [A propos d'une nouvelle
} 
l'empereur français, prenant un peu à rebours l'approche habituelle qui s'intéresse surtout à l'homme politique.

Ye Gongchao propose, lui, un compte rendu d'un ouvrage des frères Goncourt qui a une trentaine d'années et est traduit en anglais dans sa version intégrale pour la première fois, alors qu'il en existe apparemment déjà une version japonaise. ${ }^{27}$ Ye souhaite voir ce texte consacré aux femmes françaises du XVIIIe siècle rapidement traduit en chinois car il y voit une alternative au féminisme venu des Etats-Unis et de Grande-Bretagne qui se répand alors en Extrême-Orient. Reprenant l'argument des frères Goncourt, il insiste sur le fait que c'est par leur talent que les femmes doivent se faire une place dans la société et que, lorsqu'elles ont du talent - comme c'était le cas en France pour un certain nombre d'entre elles au XVIII" siècle - celui-ci est reconnu et pris en compte quel que soit le domaine concerné.

Liang Yuchun se fait, pour sa part, rapporteur de la traduction en anglais du Journal de voyage en Italie de Montaigne, texte dont il recommande la lecture non pour sa valeur littéraire (celuici a, en partie, été dicté à un secrétaire en italien) - bien qu'il présente Montaigne comme l'ancêtre et un maître de la "composition brève" (xiaopinwen 小品文) - mais pour ce que ce texte nous montre de l'homme, de son attitude face à la vie et à la société qui lui est contemporaine. .8 II le recommande, par ailleurs, à qui voudrait faire des recherches sur la période. Cet intérêt pour la vie des écrivains, et notamment pour ceux qui ont écrit des journaux, comme, par exemple, Katherine Mansfield, se retrouve à plusieurs reprises dans la revue. L'accent porte en général sur la façon dont ils parviennent à vivre leur vie d'artiste dans un monde où ils sont en marge de la société.

L'amant de Lady Chatterley représente un cas particulier car ce roman anglais, interdit par la censure au Royaume-Uni à sa sortie (1928), est présenté par Li Chendong à partir d'une version française de l'ouvrage (publiée en 1932), version sur le point d'être, elle aussi, interdite en France. C'est l'occasion, pour Li, d'évoquer les débats suscités en France par cet ouvrage sur les rapports entre littérature et morale, thème repris dans la revue par ailleurs. ${ }^{29}$ Li défend fermement l'autonomie de l'art par rapport à la morale : on ne peut pas condamner l'œuvre en raison de la morale - ou de l'absence de morale - de son auteur.

Une figure se distingue particulièrement dans ce panorama littéraire français, c'est celle d'André Maurois, avec un long article consacré au Cercle de famille (roman de Maurois publié en 1932), la traduction de deux de ses conférences, traitant, l'une, de l'écriture romanesque et, l'autre, de la passion ainsi qu'une mention dans un article traitant de la biographie littéraire. ${ }^{30}$ Le cercle de famille retrace l'histoire d'une fillette qui se rend compte de l'infidélité de sa mère, apprend à l'âge adulte que son père a, lui aussi, une maîtresse

de Napoléon récemment découverte], II.3. L'existence de cette nouvelle, vraisemblablement rédigée vers 1795, a été rendue publique dans les années 1920 et publiée intégralement en France en 1955 seulement. D'après le Dictionnaire biographique des auteurs, Tome III, Paris, Robert Laffont, 1980 (rééd.)，p，501，27 Ye Gongchao 葉公超 (19041981), "The Women of Eighteenth Century by E. \& J. Goncourt (trans. by J. Clercq and R. Roeder, Allen and Unwin Ltd.)", Xinyue, I.9, 28 Liang Yuchun, "The Diary of Montaigne's Journey to Italy in 1580 and 1581 (trans. and ed. by E. J. Trechman, Harcourt, Brace \& Co.)", II.6/7. 29 Liang Shiqiu梁實秋 aborde à plusieurs reprises le sujet N. «Wenren you xing” 文人有行 [L'homme de lettres a une règle de conduite] 1.2 ; “Wenren zhi xing " 文人之行 [La conduite de l'homme de lettres] 1.9; «Wenxue yu daode» 文學與道德 [Littérature et morale] II.8). 30 Li Chendong 李辰 冬 (1907- ), "Jiating ji gita» 家庭及其他 [Famille et autres], IV.7. Li a fait des études en France mais il est aussi un grand amateur de littérature en général et de cinéma (Etats-Unis, France, Allemagne). Dans ses articles, il a tendance à transmettre, outre une œuvre, aussi une image du pays étranger et en particulier de sa mentalité telle qu'il la perçoit. II fait davantage partager ses opinions personnelles que d'autres collaborateurs de la revue (comme Liang Yuchun ou Ye Gongchao) et, dans ses appréciations, l'aspect technique du roman passe au second plan. A ce titre, on peut dire qu'il joue un rôle important dans la réception des cultures étrangères en Chine ; Li Chendong, «Women weihe he ruhe xie xiaoshuo» 我們為何和如何寫小說 [Pourquoi et comment nous écrivons des romans], IV.5, Zeng Juezhi 曾 
(mais continue à en vouloir à sa mère) et connaît finalement la même aventure. Li Chendong en profite pour généraliser sur la pratique de l'infidélité au sein des couples français, et plus largement occidentaux, infidélité qu'il attribue à la pratique des mariages d'intérêt. Des questions sociales et politiques sont à l'occasion aussi évoquées - comme l'occupation de la Chine par les puissances étrangères et les événements qui se déroulent en URSS, thèmes abordés par Maurois dans son roman.

Mais ce qui intéresse le plus Li, c'est la conception mauroissienne du roman, et ce sur deux plans. D'abord dans le rapport à la réalité, Maurois s'inspire en effet dans ses romans de faits et d'êtres réels, mais il ne les prend que comme source d'inspiration et point de départ du roman et non comme modèles. Par ailleurs, Maurois pense que le roman n'est pas le lieu où développer des théories - celles-ci sont du ressort de l'essai - car cela nuit à l'esthétique de l'œuvre. Li adhère à cette approche, dans laquelle on peut assez facilement voir une critique adressée aux principaux promoteurs de la littérature de propagande qui commence alors à se développer en Chine.

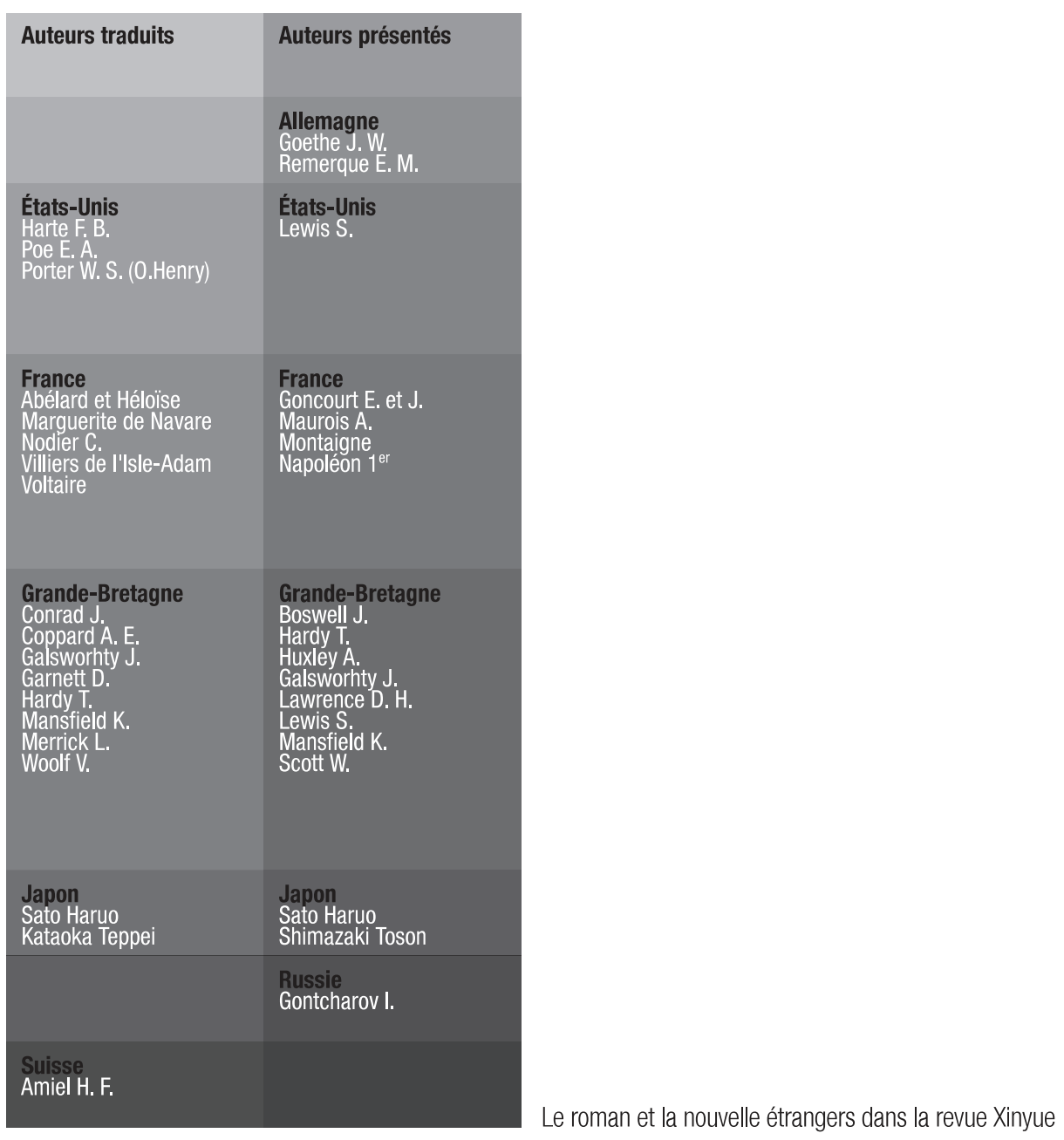


Dans le domaine poétique, c'est très largement le monde anglo-saxon qui domine dans la revue. Sur vingt-quatre articles (essais ou présentations d'œuvres), quatre seulement ne traitent pas de poésie anglaise ou américaine, dont deux sont consacrés à la France.

Dans le domaine de la traduction, ce sont au total quatorze auteurs qui sont traduits avec quarante-et-un poèmes. La France est présente avec quatre poètes : François Villon, Charles

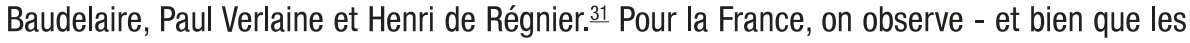
traducteurs ne soient pas les mêmes que dans le domaine du roman - une même tendance à traduire des auteurs ayant vécu à des époques différentes, le XV siècle avec Villon, le XIX ${ }^{\mathrm{e}}$ avec Baudelaire, la fin du XIX avec Verlaine et le début du XXe avec Régnier. La présence importante de Baudelaire, celles de Verlaine et de Régnier correspondent à l'engouement ressenti par les jeunes poètes chinois pour ceux qu'ils ont placés dans l'école symboliste française..$\underline{32}$ Mais aucun des poètes chinois influencés par le symbolisme français (comme Li Jinfa, Mu Mutian, Wang Duqing ou Feng Naichao) ne fait partie des poètes de la revue Xinyue. L'intérêt manifesté pour ces poètes (comme pour Villon) dans la revue Xinyue fait donc partie d'une tendance générale observable par ailleurs dans le monde littéraire chinois.

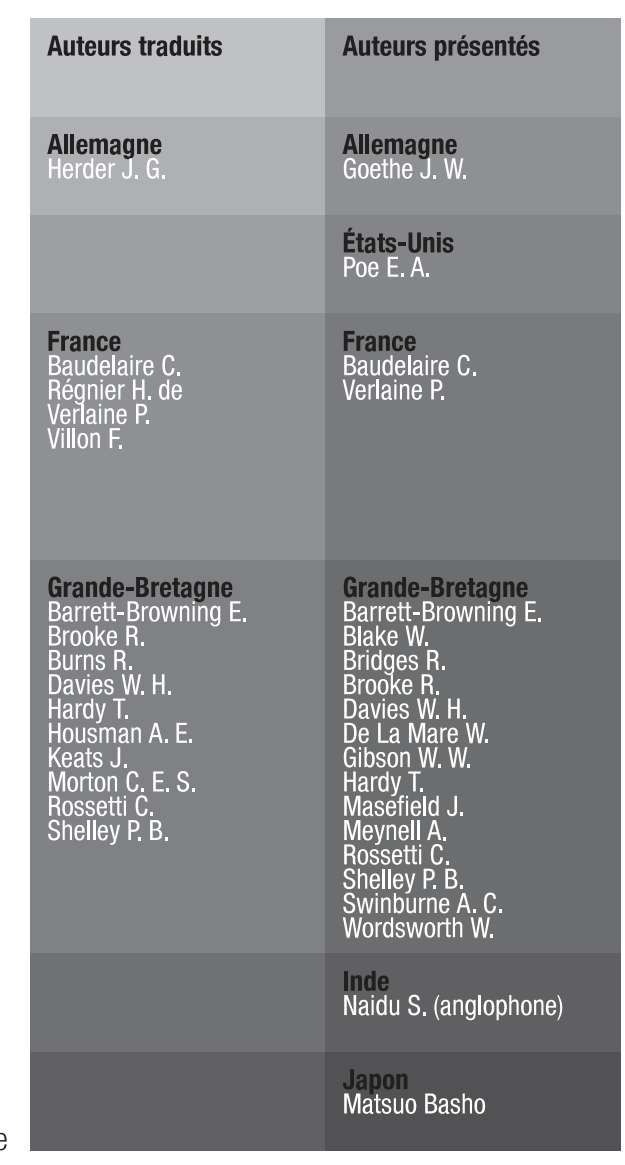


Deux essais traitent de la poésie française : l'un original, consacré à Baudelaire, par l'un des plus prestigieux collaborateurs de la revue, Xu Zhimo ; l'autre étant une traduction de Nicholson sur Verlaine.

Le bref texte de Xu Zhimo consacré aux poèmes en prose de Baudelaire témoigne de l'intérêt que le poète chinois éprouve toujours pour le poète français auquel il avait déjà consacré une traduction et un commentaire remarqués en 1924. ${ }^{33}$ Dans ce texte, Xu évoque les thèmes abordés par Baudelaire dans ses poèmes en prose, et notamment celui de son rapport à la misère des hommes et à la ville. Ce que Xu souligne, c'est avant tout l'art avec lequel Baudelaire parvient à faire éprouver de la compassion pour des êtres ou des faits qui laisseraient indifférents ou seraient jugés repoussants hors de ce contexte esthétisant. Comme chez Li Chendong lisant Maurois, c'est la question du rapport à la réalité et à sa représentation, parfois troublante, qui interpelle Xu.

Bian Zhilin propose, pour sa part, une traduction des trois derniers chapitres d'un ouvrage de Harold Nicholson intitulé Verlaine (1920). ${ }^{34}$ La traduction comporte une préface et donne, ainsi, de nombreuses informations sur la réception à cette époque du symbolisme et de Verlaine en Chine. Verlaine XE "Verlaine, Paul" est alors assez connu, ses poèmes courts ayant déjà fait l'objet de plusieurs traductions (notamment par Bian Zhilin); Bian note que ceux-ci conviennent particulièrement au goût chinois. Le symbolisme $a$, en tant que mouvement, déjà été évoqué en Chine mais Bian ne fait pas mention d'études notables. S'il a choisi ce texte de Nicholson qui porte sur les caractères intime et suggestif présents dans la poésie de Verlaine, c'est parce qu'il pense que ces deux caractéristiques se retrouvent dans la poésie chinoise traditionnelle et que ce texte montre leur fonctionnement tant dans la poésie de Verlaine XE "Verlaine, Paul" que dans la poésie chinoise, ce qui lui permet d'établir un parallèle entre les deux pratiques.

La présence de la France a évolué au fil du temps dans la revue et résulte de l'apparition de personnalités nouvelles (en particulier Li Qingya, Liang Yuchun, Li Chendong et Bian Zhilin) parmi les collaborateurs, reflétant une ouverture du groupe fondateur de la revue. Bien que les œuvres présentées ou traduites apparaissent de ton et de périodes très diverses, elles servent toujours de base à une discussion soit de questions littéraires qui sont au cœur de la réflexion des auteurs, soit de problèmes de société d'actualité en Chine.

\footnotetext{
覺之 (1901-1982), «Lian'ai de guoqu yu jianglai»戀愛的過去與將來 [Du passé et de l'avenir de la passion], III.4. Maurois est évoqué en tant que biographe aux côtés de Giles Lytton Strachey et d'Emil Ludwig. V. Liang Yuchun, «Xin zhuanii de wenxue tan» 新傳記文學譚 [Les nouvelles tendances de la biographie littéraire], Xinyue, ll.3, 31 L'Allemagne est évoquée au travers d'une traduction de J. G. Herder. Tous les autres poètes sont anglais : Elizabeth Barrett-Browning, Robert Burns, Thomas Hardy, Alfred E. Housman, Christina Rossetti, Percy B. Shelley et John Keats. Les traducteurs en sont : Bian Zhilin 市之林 et Liang Zhen 梁鎮 pour la France et l'Allemagne, Wen Yiduo, Liang Shiqiu, Rao Mengkan, Xu Zhimo et Li Weijian 李惟建 pour l'Angleterre. Liang Zhen ne publie dans la revue que des traductions de poèmes, il a étudié sous la direction de Wen Yiduo à l'Université centrale de Nankin (Zhongyang daxue). Ses centres d'intérêt sont divers, englobant notamment la France et l'Allemagne, 32 Sur la réception de Baudelaire et Verlaine en Chine, voir Jin Siyan, La Métamorphose des images poétiques 1915-1932, pp. 163-66. 33 «Botelai de sanwen shi» 波特萊的散文詩 [Les Poèmes en prose de Baudelaire], Xinyue, II-10, 34 Bian Zhilin市之林 (1910-2000), «Weierlun yu xiangzhengzhuyi»魏爾倫與象征主義 Verlaine et le symbolisme], Xinyue, IV-4. Bian Zhilin a tout juste dix-huit ans lorsque parait la revue Xinyue. II achèvera ses études à Beida quand elle disparaîtra. Sa contribution à la revue - tardive puisqu'il n'apparaît que dans le quatrième volume - se fait dans le domaine poétique et concerne la France. Bian, qui apparâtt comme un élément marginal par rapport au groupe Xinyue, montre dans son approche de la poésie symboliste une communauté d'esprit avec la plupart des membres du groupe. Ceux-ci, en se penchant sur les littératures et civilisations étrangères, se préoccupent en effet aussi de défendre leur propre culture. Bian est, par ailleurs, l'auteur des traductions de Baudelaire.
} 


\section{Une France scientifique les chercheurs français et la Chine}

Deux textes sont consacrés dans la revue à la sinologie française, tous deux rédigés par Li Huang..$\frac{35}{5}$ Celui-ci ne fait pas vraiment partie de l'entourage de la revue Xinyue et les articles qu'il publie devaient plutôt l'être dans une autre revue qui a disparu ; néanmoins, il a été en contact avec Wen Yiduo et Yu Shangyuan à leur retour de Chine en 1925 et ils se sont joints alors dans des actions de protestation contre les tentatives colonialistes des puissances occidentales. C'est la traduction de divers ouvrages d'introduction à l'histoire de disciplines comme la philosophie (Bergson), la sociologie (Durkheim) ou la sinologie (Chavannes) qui a amené Li Huang à s'intéresser à Marcel Granet et à la sinologie française.

Le texte consacré à l'histoire de la sinologie française se base sur un ouvrage d'Edouard Chavannes, rédigé à l'occasion de l'Exposition universelle de 1915 sur commande du gouvernement français (La sinologie). L'histoire de la sinologie française y est divisée en trois périodes : 1728-1783, période de familiarisation avec le monde chinois au travers de lectures et traductions ; 1815-1890, période d'approfondissement et de mise en place des bases nécessaires pour la suite, en particulier dans le domaine linguistique ; 1890-1915, début d'un véritable travail de recherche qui se traduit notamment par de bonnes interprétations et traductions des Classiques chinois en français.

Dans l'autre texte, Li propose une présentation du travail de Granet, réalisée à partir de l'ouvrage Danses et légendes de la Chine ancienne (1926), dans lequel le sinologue français expose abondamment sa méthodologie. Après une introduction consacrée à la façon dont la Haute Antiquité chinoise est abordée par les sociologues et les historiens, Li Huang fait un compte rendu de l'ouvrage de Granet puis explicite sa méthodologie. Cette relation faite sur un ton neutre se veut avant tout didactique, il s'agit de montrer aux chercheurs chinois comment ils peuvent aborder d'une autre façon l'histoire de l'Antiquité chinoise, en modifiant en particulier leur attitude face aux textes.

Les deux textes se caractérisent par une volonté didactique retenue. Li Huang semble se poser en observateur impartial, il n'interfère pas avec les arguments présentés et se contente de les exposer, semblant vouloir aborder la sinologie française de façon absolument neutre. Mais cette neutralité, qui veut donner un accès à l'objet réel de l'étude, le place en même temps dans un monde désincarné, qui semble dépourvu d'interactions avec l'autre, ce qui apparaît d'autant plus curieux que l'auteur est chinois et parle de la façon dont des étrangers étudient la Chine. Mais c'est peut-être de se sentir touché de si près qui a engendré précisément cette mise à distance alors que, dans les autres types d'articles, la distance existe d'elle-même.

35 Li Huang 李璜 (1895-1991) publie dans la revue sous le pseudonyme de Youchun 幼春/幼椿, «Faguo zhinaxuezhe Gelale de zhexue fangfa» 法國支那學格拉勒的治學方法 [La méthodologie du sinologue français M. Granet], II.8 ; «Faguo zhinaxue xiaoshi» 法國支那學小史 [La sinologie française], II.9. 


\section{Les Français vus de Chine}

Peu de textes s'intéressent dans la revue aux Français en tant que peuple, et c'est plutôt au détour d'un autre thème que la question est abordée, comme, par exemple, dans le texte de Yu Nanqiu consacré à Danton. $\frac{36}{}$ Le portrait le plus détaillé qui soit proposé des «Français » l'est dans un texte consacré aux salons littéraires du XVII' siècle. Dans celui-ci, Zhao Shaohou, l'auteur, affirme :

Les Français aiment vivre ensemble, en communauté. II est dans leur nature d'apprécier l'humour et de savoir parler, ce pour quoi ils sont particulièrement doués. Les Allemands, timides, n'osent pas s'exprimer. Les Italiens, emportés par leurs sentiments, parlent sans réfléchir. Les Anglais, trop snobs, sont mal à l'aise lorsqu'il s'agit de discourir. Et les Chinois, trop imprégnés des leçons du passé sur les risques à trop parler, eux aussi, se retiennent. En vérité, seuls les Français profitent vraiment du plaisir de la langue. II n'y a qu'eux qui parlent bien, qui soient capables de parler dix heures en une journée sans être fatigués et de serrer autant de mains [...] Leurs paroles sont comme le vol des oiseaux, sinueuses, une fois en haut, une fois en bas, une fois à droite, une fois à gauche, elles sautent d'une pensée à l'autre, du sujet le plus profond au plus léger. [...] Dans les livres, tout est discussion légère, ils discutent avec gravité des choses légères et avec légèreté des choses graves. Ils peuvent parcourir un long chemin pour arriver devant la porte d'un philosophe mais, une fois là, ils entrent, font un tour et repartent. Et parce qu'ils aiment parler, ils ne peuvent vivre seuls, hors de la société,,$\underline{37}$

C'est donc l'image d'un homme à l'esprit vif, sociable, libre, aimant plaisanter et parler, au risque de la superficialité, qui se dessine. Les femmes, Iorsqu'elles sont évoquées en tant que femmes françaises, sont intelligentes, cultivées, indépendantes et savent s'affirmer avec charme. Les origines de ces représentations sont variées : d'une part, elles semblent s'inspirer assez abondamment des œuvres et des auteurs présentés, donc de la littérature, en particulier pour ce qui est des femmes (il est essentiellement question de femmes de lettres dans la revue), mais, en même temps, le contexte dans lequel les «Français " sont définis n'est pas forcément littéraire et la représentation semble se construire, dans ce cas, en mêlant imaginaire chinois pré-existant, imaginaire individuel et expérience personnelle (les collaborateurs de la revue qui parlent de la France ont pour la plupart un rapport étroit avec elle et parlent le français).

\footnotetext{
36 Voir supra, chapitre 2: une France politique. Yu Nanqiu décrit les Français comme «des êtres libres, ayant l'esprit vif, sachant parler, tenaces, aimant la plaisanterie, nationalistes par habitude et, dernière caractéristique, se mettant facilement en colère », Voir. Yu Nanqiu, «Dantang》 丹塘 [Danton], III.5/6, 37 Zhao Shaohou 趙少侯 (18991978), «Shiqi shiji de faguo shalong» 十七世紀的法國沙龍 [Les salons français du XVI|| siècle], IV-3. Zhao est un spécialiste de la France qui a fait des études de français à Beida et a traduit, notamment, Molière, Anatole France, Alphonse Daudet et Edmond About. II a également rédigé une biographie de Romain Rolland. A partir de 1949, il est responsable des publications en français des éditions « Littérature du peuple ». Extrait traduit par l'auteur de l'article. 38 Voir supra note 4.
} 


\section{Conclusion}

De cet ensemble de textes, il ressort que la France est un pays possédant une longue tradition dans le domaine des lettres et que les femmes y occupent une place de choix, que ce soit en tant qu'auteures ou actrices de la vie littéraire (Les lettres d'Abélard et Héloïse sont le texte le plus ancien présenté). C'est également le pays des idées, qu'il s'agisse de la philosophie - même si c'est surtout un passé révolu qui est évoqué - ou de la recherche - abordée au travers de l'histoire de la sinologie. C'est aussi le pays de l'action, celui où les idées sont mises en pratique au travers, en premier lieu, de la Révolution française. C'est, enfin, un peuple avec ses particularités, peuple à la fois proche (par les problèmes qui lui sont posés) et lointain (par sa façon d'être au monde) du peuple chinois.

Les choix effectués, quant aux types de textes présentés, aux sujets abordés et aux époques concernées, portent quasiment tous à faire de la France dans la revue Xinyue, un monde rêvé, référent lointain, investi et investissable de désirs variés, renvoyant surtout, tout compte fait, à la Chine. II s'agit ici d'une France idéalisée, à la limite de l'imaginaire, servant de métaphore à la Chine. Ce n'est donc pas un hasard si la France contemporaine se résume dans la revue à une correspondance envoyée de Paris, dans laquelle les principales caractéristiques de la capitale française sont illustrées par de nombreux extraits de poèmes chinois classiques, dans la volonté sûrement très sincère, de faire appréhender la réalité de Paris aux lecteurs - qui auront sans doute trouvé Paris bien chinoise mais qui auront, tout de même, une idée de Paris, celle que s'en est faite un de leurs compatriotes. ${ }^{38}$ Au-delà de ses aspects anecdotiques, cette représentation de la France renvoie donc à l'appréhension que nous avons, en tant qu'êtres humains, de la réalité ainsi qu'à notre façon de l'investir. 